\title{
Identification of oligosaccharyltransferase as a host target for inhibition of SARS-CoV-2 and its variants
}

\author{
Yi-Jiao Huang ', Hui Zhao', Xun Huang ${ }^{1}$, Yong-Qiang Deng (1)', Xiao-Feng Li', Qing Ye ${ }^{1}$, Rui-Ting Li', Yan-Peng Xu (D)', \\ Tian-Shu Cao ${ }^{1}$ and Cheng-Feng Qin (1),2区
}

Dear Editor,

According to the report of WHO (https://covid19.who. int/), COVID-19, caused by the pandemic pathogen, severe acute respiratory syndrome coronavirus 2 (SARSCoV-2), has led to over 250 million confirmed cases and at least 5 million deaths, as of November 2021. Vaccine administration is currently the most effective way to control the COVID-19 pandemic, while novel variants of SARS-CoV-2 with concerning mutations have thrived throughout the world. Many of these variants have been evidenced to enhance viral transmissibility, fitness, infectivity, and even evade protection from vaccines ${ }^{1}$. Thus, there is an urgent need for developing effective antiviral drugs against SARS-CoV-2. So far, a large panel of antiviral agents showed promising efficiency against SARS$\mathrm{CoV}-2$ in either preclinical studies or clinical trials ${ }^{2-4}$.

Targeting host proteins associated with SARS-CoV-2 represents an alternative strategy to antagonize the emerging variants. Therapies that target the host-virus interface, which is relatively conserved between reported SARS-CoV-2 strains, could present broad-spectrum antiviral potentials ${ }^{5,6}$. Some studies have mapped the SARS-CoV-2-host interactome ${ }^{5,7}$, however, it remains a challenge to systematically explore the critical host proteins that have been already or potentially targeted by drugs.

The genome of SARS-CoV-2, which is about $29.8 \mathrm{~kb}$ in size, comprises two flanking untranslated regions and 14 open reading frames (ORFs) that encode 27 proteins, and shares $\sim 80 \%$ nucleotide sequence identity with SARS-

\footnotetext{
Correspondence: Cheng-Feng Qin (qincf@bmi.ac.cn)

${ }^{1}$ State Key Laboratory of Pathogen and Biosecurity, Beijing Institute of

Microbiology and Epidemiology, Academy of Military Medical Sciences, Beijing, China

${ }^{2}$ Research Unit of Discovery and Tracing of Natural Focus Diseases, Chinese Academy of Medical Sciences, Beijing, China

These authors contributed equally: Yi-Jiao Huang, Hui Zhao, Xun Huang.
}

$\mathrm{CoV}^{8}$. SARS-CoV-2 contains four structural proteins, spike (S), envelope $(E)$, membrane $(M)$, and nucleocapsid $(\mathrm{N})$ proteins $^{8}$. The spike, located on viral surface, is constituted by the homotrimers of $\mathrm{S}$ protein, and responsible for the recognition of host cell receptor(s) ${ }^{9}$. M protein, containing three transmembrane domains, can shape the virions and promote membrane curvature to facilitate the binding with $\mathrm{N}$ proteins, which bind virus RNA genome through different mechanisms ${ }^{9}$. E protein executes an important role in the assembly and release of virus, and is implicated in modulating viral pathogenesis ${ }^{9}$. Since these structural proteins play critical roles in the virion assembly and infection of SARS-CoV- $2^{9}$, we screened the host factors that interact with these proteins. We found that the oligosaccharyltransferase (OST) complex is closely associated with $\mathrm{E}, \mathrm{M}$ and $\mathrm{S}$ proteins, and blockade of OST by NGI-1 can significantly inhibit the infection of both SARS-CoV-2 and its variants.

To identify the potential host factors that interact with SARS-CoV-2 structural proteins, we first synthesized codon-optimized virus cDNAs of $\mathrm{E}, \mathrm{M}, \mathrm{S}$ and N proteins, and cloned these ORFs into mammalian expression vectors. The expressed structural proteins contain an $\mathrm{N}$-terminal $3 \times$ Flag tag and a C-terminal Twin-Strep-tag. We next expressed these structural proteins in HEK293T, and screened the interacting host proteins by affinitypurification-mass spectrometry (AP-MS). We found that $\mathrm{E}, \mathrm{M}$ and $\mathrm{S}$ proteins can associate with many $\mathrm{N}$ Glycosylation-related proteins, especially the OST complex, which can catalyze the $\mathrm{N}$-linked glycosylation of newly synthesized proteins ${ }^{10}$ (Fig. 1a). In addition to E, M, $\mathrm{S}$ and $\mathrm{N}$ proteins, we also detected the interacting proteins of another SARS-CoV-2-derived protein, 3a, which has been reported as a potential structural glycoprotein in SARS-CoV ${ }^{11}$, similar to SARS-CoV-2 M protein. Several $\mathrm{N}$-Glycosylation-related proteins can interact with 3a 


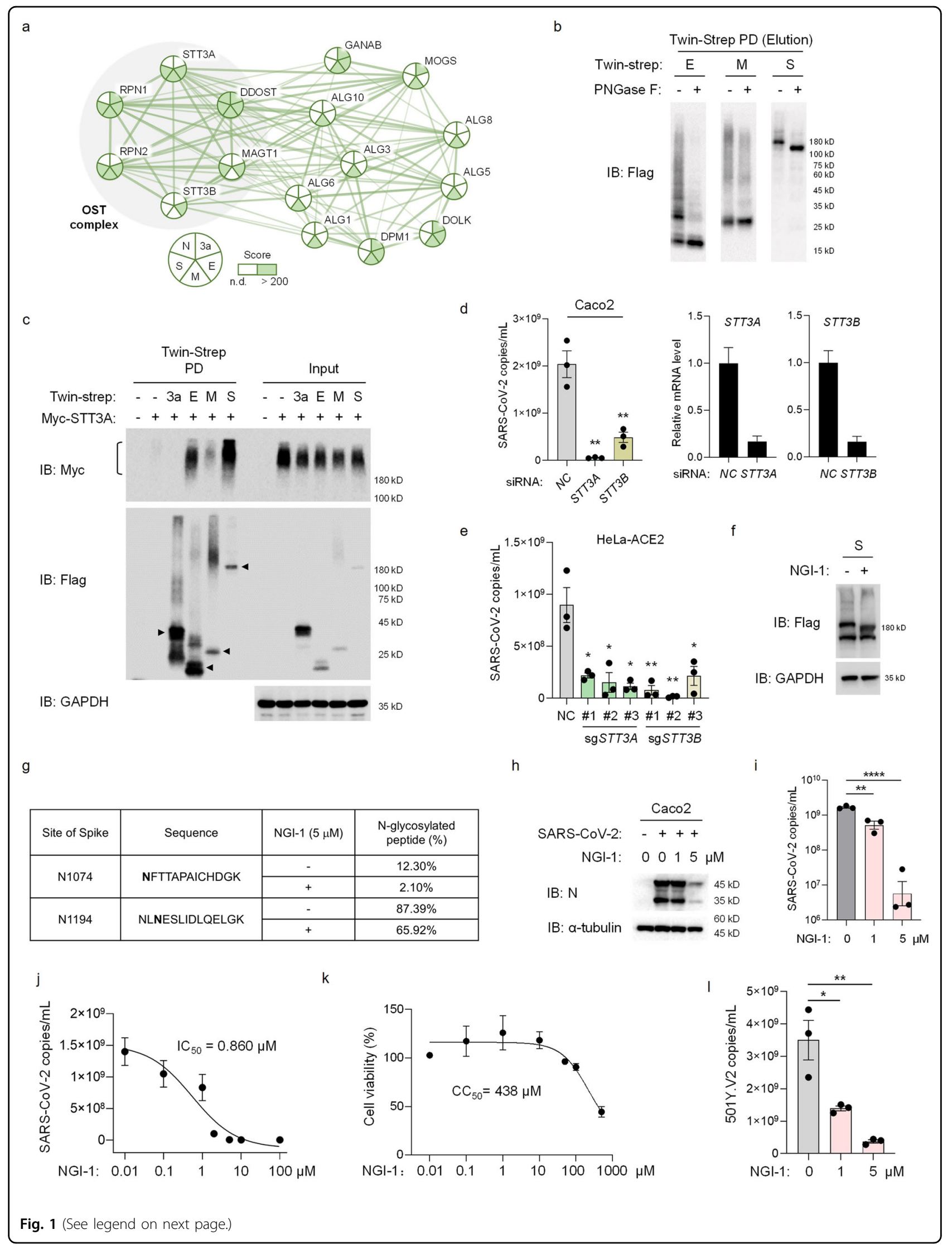


(see figure on previous page)

Fig. 1 NGI-1 inhibited SARS-CoV-2 by OST. a Biological network cluster of N-linked glycosylation according to AP-MS analysis. The original score of identified host proteins interacting with viral proteins was indicated by color scale within the circle. Score > 200 indicated sufficient confidence. n.d., not detected. The pie chart was divided into 5 slices, representing 3a, E, M, S and N proteins of SARS-CoV-2, respectively. The network was delivered according to STRING website (https://string-db.org/). Network in the gray shaded area showed OST complex. b 3x Flag-Twin-Strep tagged E, M or S proteins of SARS-CoV-2 were overexpressed in HEK293T. Proteins were pulled down after ultrasonication and eluted for further PNGase F treatment. Immunoblot analysis of virus proteins with Flag antibody. c Myc-STT3A and 3x Flag-Twin-Strep tagged 3a, E, M or S proteins of SARS-CoV-2 were overexpressed in HEK293T cells, followed by Twin-Strep pull-down. STT3A was analyzed with Myc antibody and viral proteins were analyzed with Flag antibody. The anti-GAPDH blots indicated loading of lanes. $\mathbf{d}$ STT3A or STT3B was knocked down in Caco2 cells for 3 days, followed by 3-day-SARSCoV-2 infection $(\mathrm{MOI}=0.01)$. The supernatant was collected to detect the SARS-CoV-2 gRNA by real-time quantitative polymerase chain reaction (RTqPCR). The cells were collected to detect mRNA levels of $S T T 3 A$ or $S T T 3 B$ by quantitative polymerase chain reaction (qPCR) (GAPDH as a reference gene). NC, negative control. e STT3A or STT3B was knocked out by 3 different sgRNAs respectively in HeLa cells stably expressing human ACE2 (HeLaACE2). After 3-day-infection, SARS-CoV-2 in the supernatant was analyzed by RT-qPCR. $\mathbf{f}, \mathbf{g} 3 \times$ Flag-tagged spike of SARS-CoV-2 was overexpressed in HEK293T cells, followed by 5 MM NGl-1 treatment for 2 days. Whole cell lysates were collected for Immunoblot analysis (f). Spike proteins were pulled down after ultrasonication and eluted for further MS (g). Spike was analyzed with Flag antibody. The anti-GAPDH blots indicated loading of lanes. The percentage of spike of the indicated sites, N1074 and N1194, was analyzed by Thermo Scientific Q Exactive ${ }^{\mathrm{TM}}$ Hybrid Quadrupole-Orbitrap ${ }^{\mathrm{TM}}$ Mass Spectrometer. Data are means \pm SD, from triplicates (technical replicates). $\mathbf{h}, \mathbf{i}$ Caco2 cells were infected with SARS-CoV-2 (MOI $=0.01)$ for 1 h, and the medium was replaced by $2 \% \mathrm{FBS}(\mathrm{v} / \mathrm{v})$ DMEM containing 0,1 , or $5 \mu \mathrm{M} \mathrm{NGI}-1$ for further 3-day treatment. SARS-CoV-2 in the supernatant was analyzed by RT-qPCR (i). Virus in cells was detected by immunoblotting with $\mathrm{N}$ protein antibody (h). The anti-a-tubulin blots indicated loading of lanes (h). $\mathbf{j}$ Caco 2 cells were infected with SARS-CoV-2 (MOI $=0.01)$ for $1 \mathrm{~h}$, followed by treatment of NGl-1 at the indicated concentrations for 3 days. Virus in the supernatant was analyzed by RT-qPCR. The $I_{50}$ was calculated by Statistical Package for the Social Sciences (SPSS) and the dose-response curve was fitted by GraghPad Prism 8. k Caco2 cells were plated in a 96-well plate and cultured in the presence of NGI-1 at indicated concentrations for 3 days. The cytotoxicity was analyzed by CellTiter $96^{\circ}$ AQueous One Solution Cell Proliferation Assay. The CC 50 was calculated by SPSS and the dose-response curve was fitted by GraghPad Prism 8. I Caco2 cells were infected with the South Africa variant of SARS-CoV-2 501Y.V2 (MOI = 0.01) for $1 \mathrm{~h}$, followed by treatment of NGl-1 at the indicated concentrations for 3 days. Virus in the supernatant was analyzed by RT-qPCR. Data are means \pm SEM, from triplicates (biological replicates), unpaired $t$-test, ${ }^{*} P<0.05$, ${ }^{* *} P<0.01,{ }^{* * * *} P<0.0001$ (d, e, i-I).

(Fig. 1a). These data suggested that E, $M$ and $S$ proteins may be $\mathrm{N}$-Glycosylated in host cells. To examine this hypothesis, we treated the pull-down samples with PNGase F, an amidase that can remove N-linked oligosaccharides from glycoproteins. As shown in Fig. 1b, after PNGase F treatment, the major spike band shifted to lower than $180 \mathrm{kDa}$ (Fig. 1b), indicating that spike is heavily N-Glycosylated, which is consistent with other studies $^{12,13}$. Different from $S$ protein, both $E$ and $M$ proteins displayed broad bands and main bands. After PNGase F treatment, the broad bands were decreased, while the main bands of $\mathrm{E}$ and $\mathrm{M}(\sim 20 \mathrm{kDa}$ and $\sim 30 \mathrm{kDa}$, respectively) were increased (Fig. 1b), suggesting that both $\mathrm{E}$ and $\mathrm{M}$ proteins may be glycosylated, while other posttranslational modification or oligomerization may also occur. There was no obvious variation observed for $3 \mathrm{a}$ and $\mathrm{N}$ proteins after PNGase F treatment (Supplementary Fig. S1a). These results indicated that $E, M$ and $S$ proteins were $\mathrm{N}$-Glycosylated, and OST complex may play an important role in mediating this post-translational modification.

There are two OST protein isoforms in mammalian cells $^{10,14}$. The two isoforms are composed of a catalytic subunit (encoded by the paralogs STT3A or STT3B) and accessory subunits ${ }^{14}$. We then examined the interaction between the structural proteins and STT3A/STT3B by pull-down assay. Among all tested proteins, three $\mathrm{N}$-Glycosylated proteins, E, $\mathrm{M}$ and $\mathrm{S}$ proteins, showed strong interaction with both STT3A and STT3B (Fig. 1c; Supplementary Fig. S1b). Blockade of either STT3A or
STT3B by siRNA or CRISPR-Cas9 sgRNA suppressed SARS-CoV-2 infection (Fig. 1d, e). These results indicated that both OST isoforms could modulate functions of $\mathrm{E}, \mathrm{M}$ and $\mathrm{S}$ proteins, and thus are critical for SARS-CoV-2 infection.

We next sought to confirm whether OST can serve as a potential target for treating SARS-CoV-2 infection. NGI1 , a small molecule that directly targets STT3A and STT3B, is an inhibitor of N-linked glycosylation with panflaviviral activity ${ }^{14}$. We first detected whether NGI-1 affected the N-glycosylation of SARS-CoV-2 E, M and S proteins. When treated with NGI-1, S protein, which was overexpressed in HEK293T, was detected at lower molecular weight, indicating that NGI-1 decreased the $\mathrm{N}$-glycosylation of spike (Fig. 1f), while $\mathrm{E}$ and $\mathrm{M}$ proteins showed no obvious decrease (Supplementary Fig. S2a). We next analyzed the modification changes of $\mathrm{S}$ protein with quantitative mass spectrometry. We detected two N-glycosylated sites of high abundance of spike, N1074 and N1194, and the proportion of N-glycosylated peptides of spike at N1074 site decreased from $12.30 \%$ to $2.10 \%$, and at N1194 site decreased from $87.39 \%$ to $65.92 \%$ (Fig. $1 \mathrm{~g})$. These results suggested that NGI-1 could decrease the N-Glycosylation of SARS-CoV-2.

Then, we investigated the antiviral effect of NGI-1 in human colorectal adenocarcinoma Caco2 cells. After 3-day infection, we examined virus replication in both the culture medium and $\mathrm{Caco} 2$ cells, and found a strong inhibition effect of NGI-1 on SARS-CoV-2 (Fig. 1h, i; Supplementary Fig. S2b). The half inhibitory 
concentration $\left(\mathrm{IC}_{50}\right)$ of NGI-1 and the $50 \%$ cytotoxic concentration $\left(\mathrm{CC}_{50}\right)$ of NGI-1 to Caco2 cells, were $0.860 \mu \mathrm{M}$ and $438 \mu \mathrm{M}$, respectively (Fig. 1j, k). The selectivity index $\left(\mathrm{SI}, \mathrm{CC}_{50} / \mathrm{IC}_{50}\right)$ was $\sim 509$. In addition, we detected the $\mathrm{IC}_{50}$ and $\mathrm{CC}_{50}$ in other SARS-CoV-2sensitive cell lines, including Huh-7, HeLa-ACE2 and Vero cells (Supplementary Fig. S2c), of which SI were about 255, 10541, and 260, respectively. Further, we showed that NGI-1 could efficiently inhibit the 501Y.V2 variant of SARS-CoV-2 (Fig. 11). NGI-1 also exhibited a significant inhibitory effect on the replication of $\mathrm{HCoV}$ 229E (Supplementary Fig. S3a, b). Taken together, these results indicated that the OST inhibitor NGI-1, can serve as a broad-spectrum anti-coronavirus drug.

In the present study, we focused on the potential therapies that target the host-virus interface, especially the interactions involving structural proteins of SARS$\mathrm{CoV}-2$. By AP-MS, we found that E, M and S proteins were all N-glycosylated, and were closely related to OSTrelated pathways in host, suggesting that OST may serve as a potential target for treating COVID-19. Indeed, we found that blockade of the catalytic subunit, STT3A or STT3B, of OST, suppressed SARS-CoV-2 infection. Consistently, an OST inhibitor, NGI-1, blocked the infections of both wild-type SARS-CoV-2 and its variant 501Y.V2 in vitro. During the preparation of the manuscript, another study in preprint also reported similar findings ${ }^{15}$. As NGI-1 targets OST in host cells, all SARSCoV-2 variants are supposed to be sensitive to NGI-1. In addition, by comparing several concerned variants with original SARS-CoV-2, we found that mutations in structural proteins, especially spike, have no effect on the existing or potential $\mathrm{N}$-linked glycosylation sites (sequons, $\left.(\mathrm{NXT} / \mathrm{S} / \mathrm{C} ; \mathrm{X} \neq \mathrm{P})^{10}\right)$. Interestingly, the SARS-CoV-2 variant 501Y.V3 contained the T20N mutation in S protein, which may generate an additional $\mathrm{N}$-glycosylation site that can be catalyzed by OST (from TRT to NRT). The biological impact of $\mathrm{T} 20 \mathrm{~N}$ mutation remains to be determined. Moreover, the appropriate SI of NGI-1 in various cell lines supports further validation in animal model and clinical trials. Finally, chemical library-based high throughput screening for other OST inhibitors should be warranted in the future.

\section{Acknowledgements}

This work was supported by grants from the National Key R\&D Program of China (2020YFC0841000, 2020YFA0707801, and 2021YFC0863300) and the National Natural Science Foundation of China (NSFC, 32000663, 82073621). C.-F.Q. was supported by the National Science Fund for Distinguished Young Scholar (81925025), and the Innovative Research Group (81621005) from the
NSFC, and the Innovation Fund for Medical Sciences (2019-12M-5-049) from the Chinese Academy of Medical Sciences.

\section{Author contributions}

C.-F.Q. conceived and supervised the project. Y.-J.H. designed the experiments and performed the affinity purification, drug treatment and cell experiments with the help from X.H., Y.P.X., and T.-S.C.; H.Z. performed the SARS-CoV-2 infection experiment with the help from Y.-Q.D., X.-F.L., and Q.Y.; Y.-J.H. and R.-T.L. performed mass spectrometry analysis; Y.-J.H. and H.Z. analyzed the data; Y.-J.H. and C.-F.Q. wrote the manuscript.

Conflict of interest

The authors have filed a patent related to the results reported in this work.

Publisher's note

Springer Nature remains neutral with regard to jurisdictional claims in published maps and institutional affiliations.

Supplementary information The online version contains supplementary material available at https://doi.org/10.1038/s41421-021-00354-2.

Received: 10 May 2021 Accepted: 11 November 2021

Published online: 30 November 2021

References

1. Plante, J. A. et al. The variant gambit: COVID-19's next move. Cell Host Microbe 29, 508-515 (2021)

2. Wang, M. et al. Remdesivir and chloroquine effectively inhibit the recently emerged novel coronavirus (2019-nCoV) in vitro. Cell Res. 30, 269-271 (2020).

3. Wang, G. et al. Dalbavancin binds ACE2 to block its interaction with SARS-CoV2 spike protein and is effective in inhibiting SARS-CoV-2 infection in animal models. Cell Res. 31, 17-24 (2021).

4. Zu, S. et al. 25-Hydroxycholesterol is a potent SARS-CoV-2 inhibitor. Cell Res. 30, 1043-1045 (2020).

5. Gordon, D. E. et al. A SARS-CoV-2 protein interaction map reveals targets for drug repurposing. Nature 583, 459-468 (2020).

6. Prussia, A., Thepchatri, P., Snyder, J. P. \& Plemper, R. K. Systematic approaches towards the development of host-directed antiviral therapeutics. Int. J. Mol. Sci. 12, 4027-4052 (2011).

7. Cava, C., Bertoli, G. \& Castiglioni, I. A protein interaction map identifies existing drugs targeting SARS-CoV-2. BMC Pharmacol. Toxicol. 21, 65 (2020).

8. Wu, A. et al. Genome composition and divergence of the novel coronavirus (2019-nCoV) originating in China. Cell Host Microbe 27, 325-328 (2020).

9. Bhat, E. A. et al. SARS-CoV-2: Insight in genome structure, pathogenesis and viral receptor binding analysis - an updated review. Int. Immunopharmacol. 95, 107493 (2021)

10. Shrimal, S. \& Gilmore, R. Oligosaccharyltransferase structures provide nove insight into the mechanism of asparagine-linked glycosylation in prokaryotic and eukaryotic cells. Glycobiology 29, 288-297 (2019).

11. Oostra, M., de Haan, C. A., de Groot, R. J. \& Rottier, P. J. Glycosylation of the severe acute respiratory syndrome coronavirus triple-spanning membrane proteins 3a and M. J. Virol. 80, 2326-2336 (2006).

12. Tian, W. et al. O-glycosylation pattern of the SARS-CoV-2 spike protein reveals an "O-Follow-N" rule. Cell Res. 31, 1123-1125 (2021).

13. Yao, H. et al. Molecular architecture of the SARS-CoV-2 virus. Cell 183, 730-738 e713 (2020).

14. Puschnik, A. S. et al. A small-molecule oligosaccharyltransferase inhibitor with pan-flaviviral activity. Cell Rep. 21, 3032-3039 (2017).

15. Casas-Sanchez, A. et al. Protein N-glycosylation is essential for SARS-CoV-2 infection. bioRxiv. https://doi.org/10.1101/2021.02.05.429940 (2021). 